\title{
Silicon Microsphere Photonics
}

\author{
Ali Serpengüzel, ${ }^{*}$ Adnan Kurt, and Ulas Kemal Ayaz ${ }^{\dagger}$ \\ Koç University, Microphotonics Research Laboratory, Physics Department, \\ Rumelifeneri Yolu, Sariyer, Istanbul 34450 Turkey
}

\begin{abstract}
Electrophotonic integrated circuits (EPICs), or alternatively, optoelectronic integrated circuit (OEICs) are the natural evolution of the microelectronic integrated circuit (IC) with the added benefit of photonic capabilities. Traditionally, the microelectronics IC industry has been based on group IV silicon, whereas the microphotonics industry on group III-V semiconductors. However, silicon based photonic microdevices have been making strands in "siliconizing" photonics. Silicon microspheres with their high quality factor whispering gallery modes (WGMs), are ideal candidates for wavelength division multiplexing (WDM) in the standard near-infrared telecommunications bands. In our experiments, we are using silicon microspheres with a refractive index of 3.48 and a radius of 500 micrometers. The optical resonances of the silicon microspheres provide the necessary narrow linewidths, that are needed for high resolution WDM applications. Potential WDM applications include filters, modulators, switches, detectors, and possibly light sources.
\end{abstract}

Keywords: channel dropping, detector, frequency selective, microsphere, morphology dependent resonance (MDR), light coupling, modulation, optical fiber coupler, silicon, silicon on oxide, silicon on insulator (SOI), waveguide, wavelength division multiplexing (WDM), whispering gallery mode (WGM).

\section{INTRODUCTION}

With the development of optoelectronics, metal interconnections are no longer limiting factor for the performance of electronic systems. Replacing the metal interconnections by optical interconnections could provide low power dissipation, low latencies, and high-bandwidths [1]. Such optical interconnections rely on the integration of microphotonics with microelectronics, thereby creating the electrophotonic integrated circuits (EPICs), or alternatively, optoelectronic integrated circuits (OEICs) [2].

\section{SILICON PHOTONICS}

Silicon has been the material of choice for the microelectronics industry for more than half-a-century [3] since it is a relatively inexpensive, and well understood material for producing complementary metal oxide semiconductor (CMOS) based microelectronic devices. Silicon is the second most abundant material on earth after oxygen, its silica partner. Additionally, the need for low cost photonic devices has stimulated a significant amount of research in silicon photonics [4]. Although silicon photonics is less well developed as compared to the direct bandgap (i.e., light emitting) III-V semiconductor photonics technologies; silicon is poised to make a serious impact on the optical communications, as well

\footnotetext{
* Correspondence should be addressed to Ali Serpengüzel, aserpenguzel@ku.edu.tr; phone: + 90 (212) 338-1312; fax: + 90 (212) 338-1547, http://home.ku.edu.tr/ aserpenguzel, http://microphotonics.ku.edu.tr, Koç University, Microphotonics Research Laboratory, Physics Department, Rumeli Feneri Yolu, Sariyer, Istanbul 34450 Turkey. ${ }^{\dagger}$ Ulas Kemal Ayaz is currently with Polytechnic University, Department of Mechanical, Aerospace, and Manufacturing Engineering, Six MetroTech Center, Brooklyn NY 11201, USA.
}

Photonic Materials, Devices, and Applications II, edited by Ali Serpengüzel,Gonçal Badenes, Giancarlo Righini Proc. of SPIE Vol. 6593, 65931J, (2007) · 0277-786X/07/\$18 · doi: 10.1117/12.724173 
as in many other microphotonic and microelectronic applications with the advent of integrated silicon optoelectronics [5] and the electro-photonic integrated circuit (EPIC) technology.

Recent progress in silicon photonics is being heralded by the observation of first the Raman gain [6,7] then stimulated Raman scattering (SRS) [8] in a crystalline silicon waveguide, SRS lasing first in pulse [9,10] modulated [11] and later in CW [12] silicon Raman lasers, and finally the hybrid silicon Raman laser [13]. Additionally, silicon modulators [14,15] have been developed first using a metal-oxide-semiconductor (MOS) capacitor [16], a Mach-Zehnder [17] configuration, SRS [18], and a microring [19] configuration.. Silicon-germanium resonant cavity enhanced (RCE) photodetectors are being developed for all silicon based electro-photonic microintegration. Recently, a silicon microring based wavelength converter has also been realized [20]. With well established CMOS processing techniques, it will be possible to integrate lasers, waveguides, modulators, switches, wavelength converters, and photodetectors into silicon motherboards [21, 22] for wavelength division multiplexing (WDM) applications [23] as well as intrachip communication applications, such as clock distribution.

\section{MICROSPHERE RESONATORS}

The three dimensional (3D) analogue of the well known two dimensional (2D) microring/microtoroid resonator, microsphere resonators are also ideal 3D microphotonic building blocks due to their small volumes and high quality factors. There have been various photonic applications of microspheres in the ultraviolet (UV), visible and near-infrared (IR) communication bands [24]. Morphology dependent resonances (MDR's), i.e., whispering gallery modes (WGM's), or simply optical resonances of microspheres provide the necessary optical feedback for applications in spectroscopy, laser science, and optical communications. Microlasers, optical couplers, and optical filters are some of the applications of microspheres. Low threshold lasing from rare earth doped silica microspheres [25], polymer microsphere lasers [26] and Raman lasers have been demonstrated. Strain tunable microsphere oscillators [27], add-drop filters [28], and thermooptical switching [29] have been realized for the frequency control in optical communications. Microsphere resonators are uniquely applicable in compact optoelectronic devices in wavelength division multiplexing (WDM) applications [30]. Recently, we have observed elastic light scattering in silicon microspheres in the near-infrared [31]. In this paper, we are investigating the electrooptical response of these silicon microspheres.

\section{MORPHOLOGY DEPENDENT RESONANCES}

A physical interpretation of MDR's is based on the propagation of rays around the inside surface of the microsphere, confined by an almost total internal reflection (TIR) [32]. The rays approach the interior surface of the microsphere at an angle beyond the critical angle and thus experience an almost total internal reflection (TIR). The light rays follow the interior surface of the microsphere, and return to their respective entrance points at the same phase and start to propagate in the interior surface of the microsphere all over again. This basically is the physical interpretation of the MDR's for a microsphere. The light, after circumnavigating the interior surface of the microsphere, returns to its entrance point and interferes with itself. At resonant wavelengths, the interference is constructive and the elastically scattered light from the microsphere reaches to peak intensities. Respective to the peaks in elastically scattered light, the intensity of transmitted light through fiber experiences associated dips in intensity [33]. MDR's satisfy resonance conditions for specific values of the size parameter $x=2 \pi a / \lambda$, where $a$ is radius of the sphere and $\lambda$ is the vacuum wavelength of light [34].

The MDR's of a microsphere are analyzed by the localization principle [35] and the generalized Lorenz-Mie theory (GLMT) [36]. The angular mode number (n) gives the number of maxima between 0 and 180 degrees in the polar angular distribution of the energy of the MDR. The radial mode order (l) indicates the number of maxima in the internal electric field distribution in the radial direction. Each MDR of the microsphere also has an azimuthal angular dependence from 0 to 360 degrees, which is labeled with an azimuthal mode number. However, for spheres, MDR's differing only in azimuthal mode number have identical resonance frequencies. The greatest impediment to the use of microsphere resonators has been the difficulty of coupling the light into and out of the microspheres efficiently. Light can be coupled into the microsphere by various types of coupling devices: side-polished optical fibers [37], prisms [38] and tapered optical fibers [39]. The principle of these devices is based on providing efficient energy transfer to the resonant circular TIR guided wave in the microsphere resonator through the evanescent field. There are two main conditions to be 
satisfied to achieve efficient coupling: phase synchronism and significant overlap of the two waves modeling the MDR mode and the coupler mode. The coupling is based on the fact that the modal field of the guided mode extends beyond the core-cladding interface.

\section{ELECRO-OPTICAL EFFECT IN SILICON}

In the presence of free carriers in silicon, both the refractive index and the absorption of silicon can be changed using carrier injection or depletion. The refractive index changes due to free carrier accumulation is given by the KramersKronig analysis [4]:

$$
\begin{aligned}
& \Delta \mathrm{n}_{\mathrm{e}}=-8.8 \times 10^{-22} \Delta \mathrm{N}_{\mathrm{e}} \\
& \Delta \mathrm{n}_{\mathrm{h}}=-8.5 \times 10^{-18}\left(\Delta \mathrm{N}_{\mathrm{h}}\right)^{0.8}
\end{aligned}
$$

where $\Delta \mathrm{n}_{\mathrm{e}}$ and $\Delta \mathrm{n}_{\mathrm{h}}$ are the changes in the refractive index resulting from change in the free electron carrier concentrations and change in the free hole concentrations. $\Delta \mathrm{N}_{\mathrm{e}}$ and $\Delta \mathrm{N}_{\mathrm{h}}$ are the free electron and free hole concentrations, respectively.

\section{ELECTRICAL MEASUREMENTS}

By placing two probes to the poles of the sphere, we obtained a metal-silicon-metal (MSM) contact. Figure 1 shows the schematic diagram of the MSM contact that was used in our experiments. The I-V characteristics illustrated in figure 2 support that the MSM contact exhibits the behavior of two Schottky diodes connected back to back. The graph is not fully symmetric, due to the non-symmetric contact. The contact resistance differences are not significant.

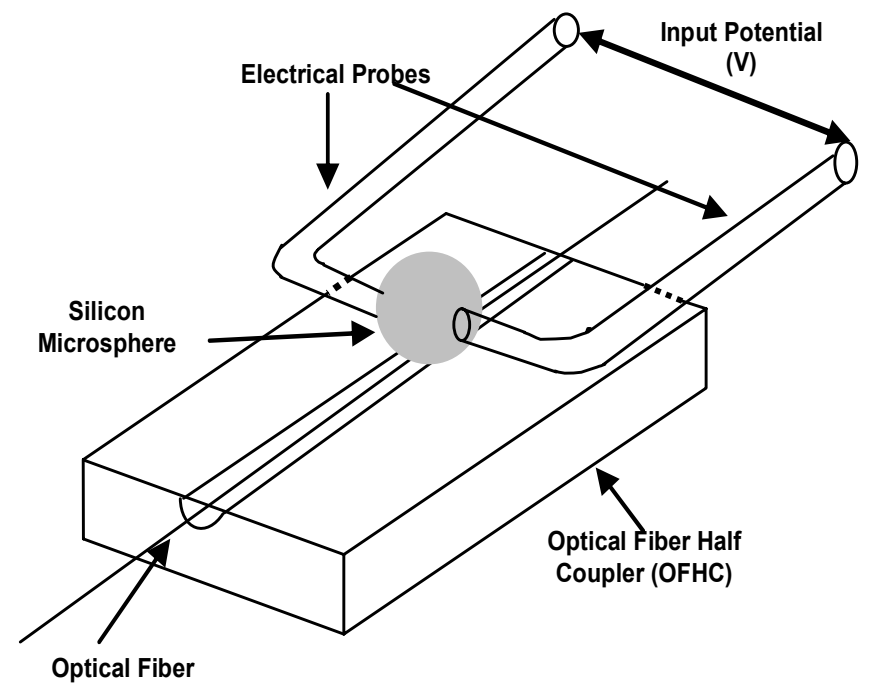

Fig.1. Illustration of electrical input to the microsphere over the OFHC. 


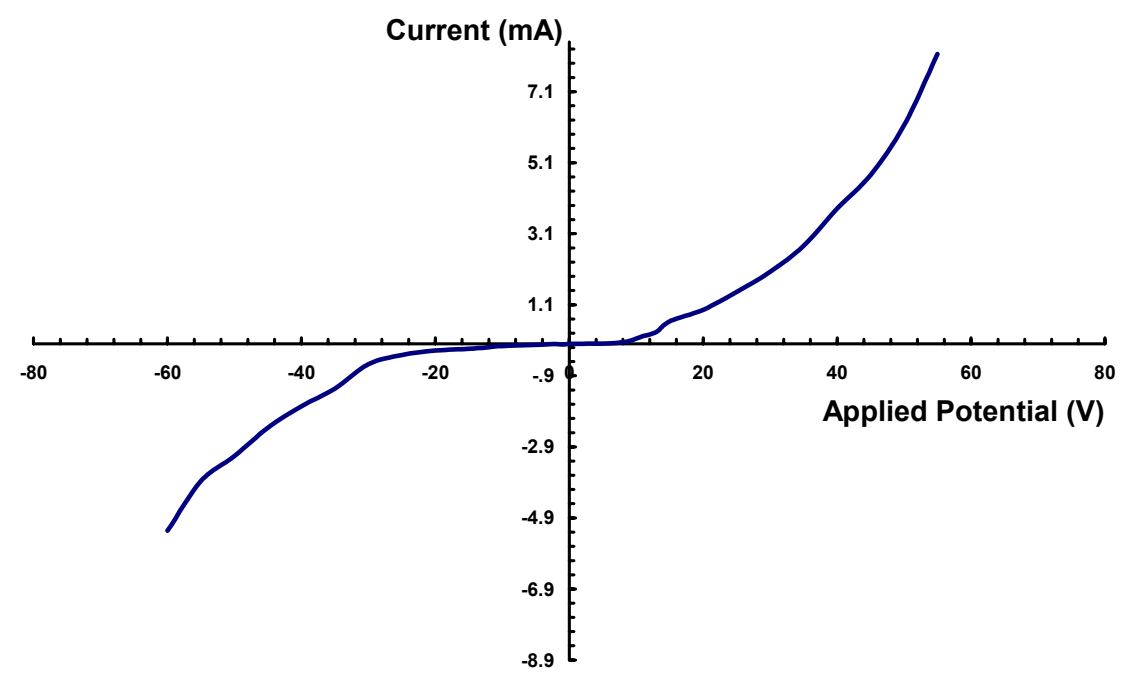

Fig.2. The I-V characteristics of the experimental metal-silicon-metal (MSM) contact.

\section{OPTICAL MEASUREMENTS}

Light is coupled to the silicon microsphere by using an optical fiber half coupler (OFHC). Figure 3 shows the optical geometry of the microsphere and the OFHC. The OFHC is manufactured by polishing the optical fiber that is glued in a silica substrate. OFHC is fabricated from $1500 \mathrm{~nm}$ standard single mode fiber (SMF). The cladding is polished down to approach the evanescent field of the optical fiber. The silicon microsphere used in the experiment has a radius a $=500$ $\mu \mathrm{m}$ and refractive index $\mathrm{n}=3.48$. The silicon microsphere is placed on the interaction region of the OFHC.

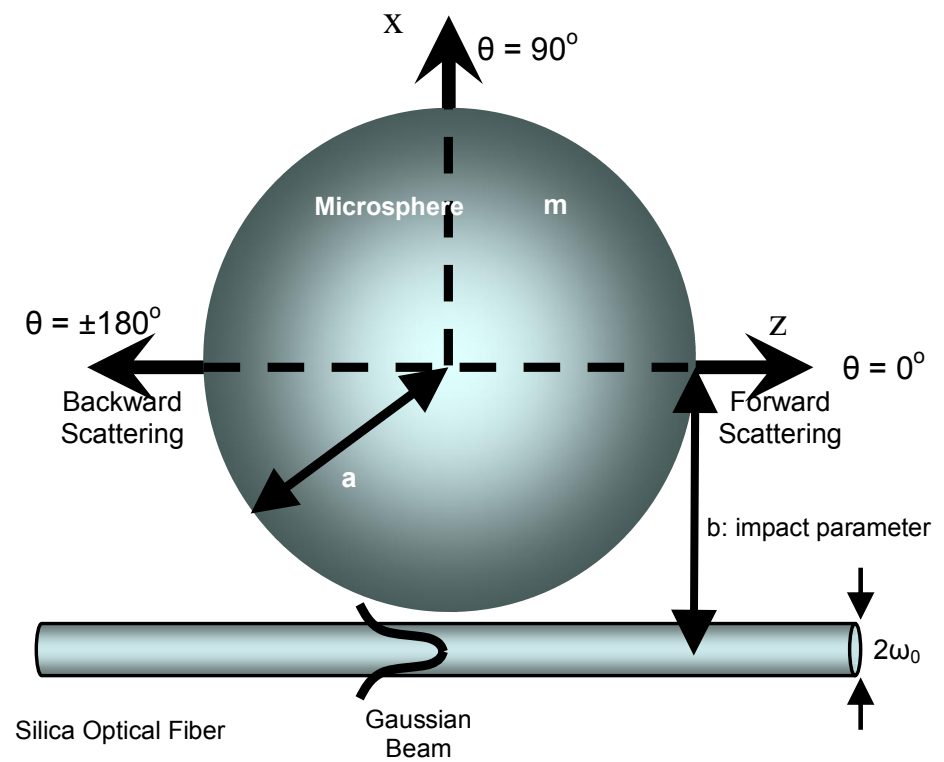

Fig.3. Schematic illustration of the optical fiber excitation of the silicon microsphere. 


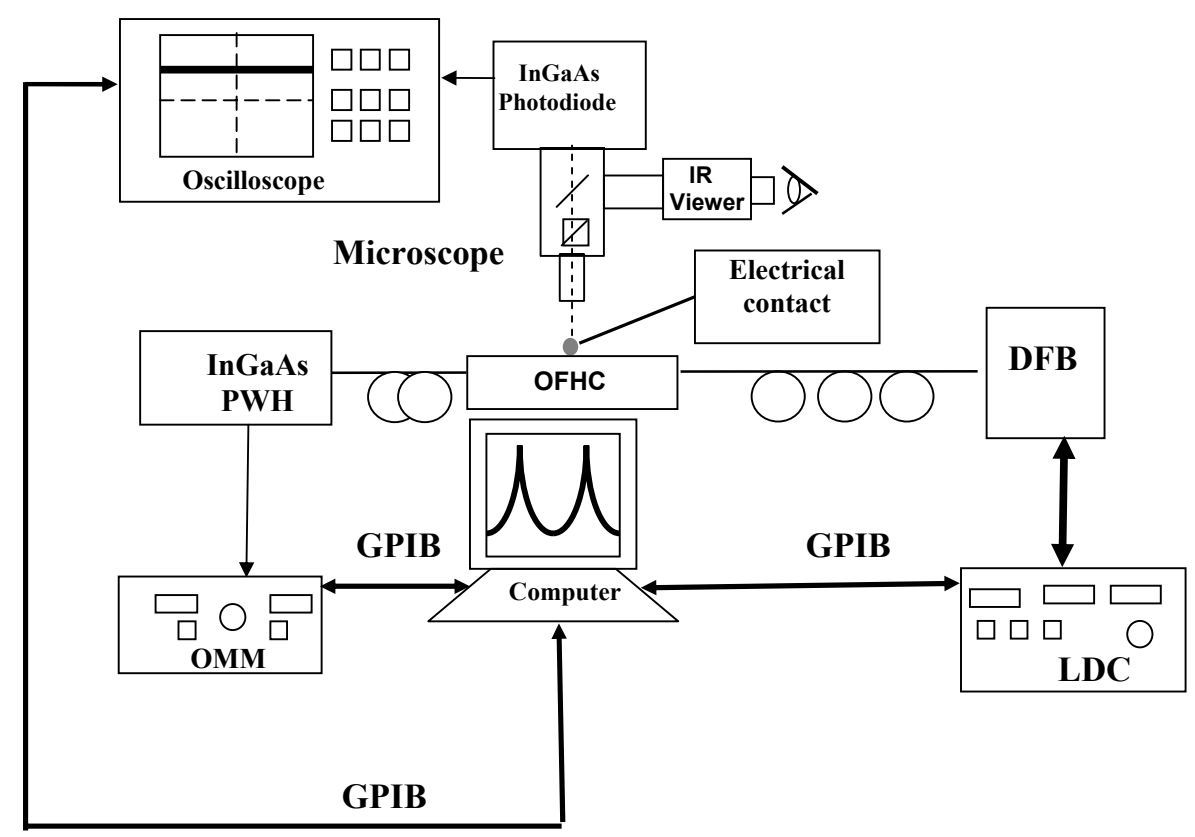

Fig.4. The schematic of the experimental setup.

Figure 4 shows the experimental setup. The output of a distributed feedback (DFB) laser is coupled to the optical fiber. Temperature tuning of the DFB laser is achieved by using a laser diode controller (LDC). The transmitted light is observed using an InGaAs optical power/wave head connected to an optical multimeter (OMM). The elastically scattered light from the microsphere is collected through a microscope objective and detected by the InGaAs photodiode. The microsphere was held in position by electrical probes from the poles, which were also used for electrical input. A typical spectrum is shown in Fig. 5.

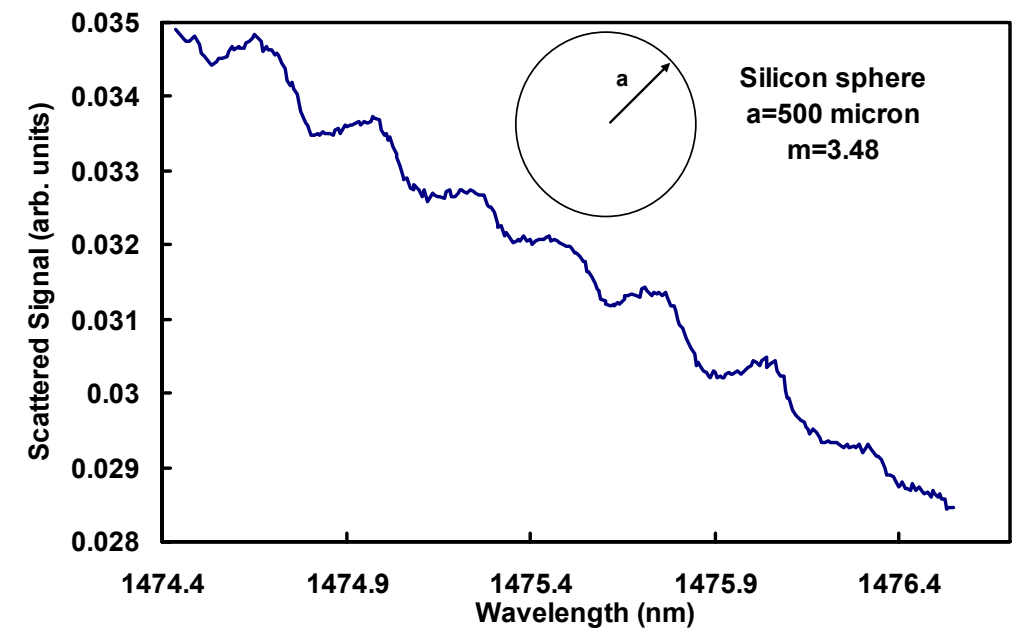

Fig.5. A typical spectrum from the silicon microsphere.

\section{CONCLUSIONS}

We have experimentally observed the elastic scattered signal from a silicon microsphere in the near-IR at a wavelength of $1475 \mathrm{~nm}$. The mode spacing $(\Delta \lambda)$, i.e. wavelength difference between consecutive mode numbers (n) with the same 
mode order (1), is measured to be $0.27 \mathrm{~nm}$. Moreover, we have measured the IV characteristics of the silicon microsphere sandwiched between metal probes. The MSM structure exhibits two back to back Schottky diode response. These observations herald novel active silicon microsphere based optoelectronic device applications. Possible wavelength division multiplexing (WDM) applications include optoelectronic devices for guiding, filtering, modulating, switching, detecting, and lasing.

\section{ACKNOWLEDGMENTS}

We would like to acknowledge the partial support of this research by the European Commission Grant No: FP6-IST511616: PHOREMOST, FP6-IST-003887 NEMO, and the conference support by the European Office of Aerospace Research and Development, Grant No:FA8655-07-1-5013.

\section{REFERENCES}

1. M. Paniccia, M. Morse, and M. Salib, "Integrated Photonics," in "Silicon Photonics," L. Pavesi and D. J. Lockwood, Eds. Springer Verlag, Berlin, 51-85 (2004).

2. G. Guillot and L. Pavesi, "Optical Interconnects: The Silicon Approach,” Springer Verlag, Berlin (2006).

3. L. Pavesi, "Will silicon be the photonic material of the next millennium?" J. Phys: Condens. Matter, 15, 1169-1196 (2003).

4. G.T. Reed and A.P. Knights, "Silicon Photonics: An Introduction," Wiley, New York (2004).

5. H. Zimmermann, "Integrated Silicon Optoelectronics," Springer Verlag, Berlin (2000).

6. A. Liu, H. Rong, M. Paniccia, O. Cohen, and D. Hak, "Net Optical Gain in a Low Loss Silicon-on-Insulator Waveguide by Stimulated Raman Scattering," Opt. Express 12, 4261-4268 (2004).

7. H. Rong, A. Liu, R. Nicolaescu, M. Paniccia, "Raman Gain and Nonlinear Optical Absorption Measurements in a Low-Loss Silicon Waveguide," Appl. Phys. Lett. 85, 2196-2198 (2004).

8. R. Jones, H. Rong, A. Liu, A. Fang, and M. Paniccia, "Net Continuous Wave Optical Gain in a Low Loss Silicon-on-Insulator Waveguide by Stimulated Raman Scattering," Opt. Express 13, 519-525 (2005).

9. O. Boyraz and B. Jalali, "Demonstration of a silicon Raman laser," Opt. Express 12, 5269-5273 (2004).

10. H. Rong, A. Liu, R. Jones, O. Cohen, D. Hak, R. Nicolaescu, A. Fang and M. Paniccia, "An All-Silicon Raman Laser," Nature 433, 292-294 (2005).

11. O. Boyraz and B. Jalali, "Demonstration of directly modulated silicon Raman laser," Opt. Express 13, 796-800 (2005).

12. H. Rong, R. Jones, A. Liu, O. Cohen, D. Hak, A. Fang, and M. Paniccia, "A Continuous-Wave Raman Silicon Laser," Nature 433, 725-728 (2005).

13. A. W. Fang, H. Park, O. Cohen, R. Jones, M. J. Paniccia, and J. E. Bowers, "Electrically pumped hybrid AlGaInAs-silicon evanescent laser," Opt. Express, 14, 9203-9210 (2006).

14. A. Liu, D. Samara-Rubio, L. Liao, and M. Paniccia, "Scaling the Modulation Bandwidth and Phase Efficiency of a Silicon Optical Modulator," IEEE J. Sel. Top. Quantum Electron. 11,367-372 (2005).

15. L. Liao, A. Liu, R. Jones, D. Rubin, D. Samara-Rubio, O. Cohen, M. Salib and M. Paniccia, "Phase Modulation Efficiency and Transmission Loss of Silicon Optical Phase Shifters," IEEE J. Quantum Electron. 41, 250-257 (2005).

16. A. Liu, R. Jones, L. Liao, D. Samara-Rubio, D. Rubin, O. Cohen, R. Nicolaescu, M. Paniccia, "A High-Speed Silicon Optical Modulator based on a Metal-Oxide-Semiconductor Capacitor," Nature 427, 615-618 (2004).

17. L. Liao, D. Samara-Rubio, M. Morse, A. Liu, D. Hodge, D. Rubin, U. D. Keil, and T. Franck, "High Speed Silicon Mach-Zehnder Modulator," Opt. Express 13, 3129-3135 (2005).

18. R. Jones, A. Liu, H. Rong, M. Paniccia, O. Cohen, and D. Hak, "Lossless Optical Modulation in a Silicon Waveguide using Stimulated Raman Scattering," Opt. Express 13, 1716-1723 (2005).

19. Q. Xu, S. Manipatruni, B. Schmidt, J. Shakya, and M. Lipson, "12.5 Gbit/s carrier-injection-based silicon microring silicon modulators," Opt. Express 15, 431-436 (2007).

20. S. F. Preble, Q. Xu, and M. Lipson, "Changing the colour of light in a silicon resonator," Nature Photon. 1, 293-296 (2007).

21. B. Jalali, S. Yegnanarayanan, T. Yoon, T. Yoshimoto, I. Rendina, and F. Coppinger, “Advances in Silicon on insulator Optoelectronics," IEEE J. Select. Top. Quantum Electron. 4, 938-947 (1998).

22. A. Kaneko, T. Goh, H. Yamada, T. Tanaka, and I. Ogawa, "Design and applications of silica-based planar lightwave circuits," IEEE J. Select. Top. Quantum Electron. 5, 1227-1236 (1999).

23. B. J. Offrein, R. Germann, F. Horst, H. W. M. Salemink, R. Beyerl, and G. L. Bona, "Resonant coupler-based tunable add-after-drop filter in silicon-oxynitride technology for WDM networks," IEEE J. Select. Top. Quantum Electron. 5, 1400-1406 (1999).

24. S. M. Spillane, T. J. Kippenberg, and K. J. Vahala, "Ultralow-threshold Raman laser using a spherical dielectric microcavity", Nature 415, 621623 (2002).

25. V. Lefèvre-Seguin and S. Haroche, "Towards cavity-QED experiments with silica microspheres", Mat. Sci. Eng. B48, 53-58, (1997).

26. M. Kuwata-Gonokami and K. Takeda, "Polymer whispering gallery mode lasers", Opt. Mat. 9, 12-17 (1998).

27. V. S. Ilchenko, P. S. Volikov, V. L. Velichansky, F. Treussart, V. Lefèvre-Seguin, J.-M. Raimond, and S. Haroche, "Strain-tunable high-Q optical microsphere resonator", Optics Comm. 145, 86-90 (1998).

28. M. Cai, G. Hunziker, and K. J. Vahala, "Fiber-optic add-drop device based on a silica microsphere- whispering gallery mode system," IEEE Photon. Technol. Lett. 6, 686-687 (1999). 
29. H. C. Tapalian, J.-P. Laine, and P. A. Lane, "Thermooptical switches using coated microsphere resonators", IEEE Photon. Technol. Lett. 14, 1118-1120 (2002).

30. A. Serpengüzel, S. Arnold, G. Griffel, and J. A. Lock, "Enhanced coupling to microsphere resonances with optical fibers," J. Opt. Soc. Am. B. 14, 790-795 (1997).

31. Y.O. Yilmaz, A. Demir, A. Kurt, and A. Serpengüzel, "Optical Channel Dropping with a Silicon Microsphere,” IEEE Photon. Technol. Lett. 17, $1662-1664$ (2005).

32. P. Barber and R. K. Chang, Optical Effects Associated with Small Particles, World Scientific Press, Singapore (1988).

33. P. Barber, and S. C. Hill, Light Scattering by Particles: Computational Methods, World Scientific Press, Singapore (1990).

34. M. Pelton and Y. Yamamato, "Ultralow threshold laser using a single quantum dot and a microsphere cavity," Phys. Rev. A 59, 2418-2421 (1999).

35. H.M. Nussenzveig, "Diffraction Effects in Semiclassical Scattering," Cambridge University Press, Cambridge (1992).

36. G. Griffel, S. Arnold, D. Taskent, A. Serpengüzel, J. Connolly, and N. Morris, "Morphology Dependent Resonances of a Microsphere, Optical Fiber System," Opt. Lett. 21, 695-697 (1996).

37. A. Serpengüzel, S. Arnold, and G. Griffel, “Excitation of Resonances of Microspheres on an Optical Fiber”, Opt. Lett. 20, 654-656 (1995).

38. M. L. Gorodetsky and V. S. Ilchenko, "High-Q optical whispering gallery microresonators: precession approach for spherical mode analysis and emission patterns," Opt. Commun. 113, 133-143 (1994).

39. J. C. Knight, G. Cheung, F. Jacques, and T. A. Birks, "Phase matched excitation of whispering gallery modes by a fiber taper," Opt. Lett. 22, 1129-1131 (1997). 